4. Cates CJ, Lasserson TJ. Combination formoterol and budesonide as maintenance and reliever therapy versus inhaled steroid maintenance for chronic asthma in adults and children. Cochrane Database Syst Rev 2009; (2):CD007313

5. Cates CJ, Lasserson TJ. Combination formoterol and inhaled steroid versus beta2-agonist as relief medication for chronic asthma in adults and children. Cochrane Database Syst Rev 2009;(2):CD007085.

\section{Single maintenance and reliever therapy}

The paper by Chapman et al ${ }^{1}$ reviewing single maintenance and reliever therapy (SMART) in asthma is important in highlighting some of the inadequacies of existing research evaluating this treatment method.

The authors also claim that SMART fails to achieve adequate asthma control as measured by GINA criteria and provide a table detailing seven studies and associated control indices.

While these outcomes are far from ideal, the authors fail to point out that they were no worse than the comparator arm, which varied across the studies from conventional inhaled steroid therapy to fixed dose combination inhaled steroid/long-acting betaagonist inhalers in high dose (ie, 'optimal therapy'). This inadequate control therefore reflects the severity of disease in the trial subject group rather than being a specific deficiency of SMART therapy.

It is disingenuous to claim that SMART fails to achieve adequate asthma control without pointing out that in this patient group standard, 'optimal', therapy does no better.

\section{Simon Bowler, David Serisier}

Department of Respiratory Medicine, Mater Adult Hospital, South Brisbane, Australia

Correspondence to Dr Simon Bowler, Department of Respiratory Medicine, Mater Adult Hospital, Suite 22, 293 Vulture Street, South Brisbane 0 4101, Australia; lungmed@mc.mater.org.au

Competing interests SB reports receiving travel assistance, speakers fees and advisory board fees from Astra Zeneca, Glaxo Smith Kline and Novartis. DS reports receiving advisory board and speakers fees from Glaxo Smith Kline and Astra Zeneca.

Provenance and peer review Not commissioned; not externally peer reviewed.

Accepted 30 August 2010

Published Online First 19 November 2010

Thorax 2011;66:87. doi:10.1136/thx.2010.150219

\section{REFERENCE}

1. Chapman K, Barnes N, Greening P, et al. Single maintenance and reliever therapy (SMART) of asthma: a critical appraisal. Thorax 2010;65:747-52. doi:10.1136/thx.2009.128504.

\section{Authors' response}

We thank our colleagues who have forwarded questions and comments to the editors of Thorax, thereby engaging in a discussion of asthma strategy we believe to be long overdue. We must leave the editors of Thorax to respond to comments directed to their principles and policies, but suspect that our review was regarded by the editors and reviewers as a summary of single maintenance and reliever therapy (SMART) outcomes from a traditional yet unexplored perspective that might spark discussion in an important area. In doing so, we believe that the journal has behaved responsibly by encouraging scientific debate. The tenets of single maintenance and reliever therapy of asthma have represented a marked departure from contemporary asthma management perspectives. These include the following: (1) that a reactive and bronchodilator-driven strategy of asthma care is superior to the prevention of asthma symptoms and disability as long as a small aliquot of inhaled corticosteroid is inhaled at times of acute wheezing and breathlessness; (2) that comprehensive asthma control is no longer needed to evaluate asthma treatment and it is sufficient to measure the time between severe exacerbations; and (3) that rising sputum and biopsy markers of inflammation are of no concern in the choice of maintenance strategies. Until the present correspondence, the absence of discussion and debate concerning these proposals has puzzled us.

Dr Peters and Professor Jenkins have entitled their letter 'Critical appraisal of Symbicort maintenance and reliever treatment misrepresents clinical evidence'. ${ }^{1}$ We had used the acronym SMART to represent 'single maintenance and reliever therapy' to engage in a broad discussion of asthma management principles and not a review of a specific pharmacotherapy; that will be the intended meaning of the acronym in this letter. ${ }^{2}$ Peters and Jenkins state that we have implied that fixed dose treatments 'achieved target levels of control' in reference to the review of control outcomes by Bateman and colleagues. ${ }^{3}$ We can find no mention of fixed dose treatment outcomes in this paragraph of our publication. Elsewhere in the review we have noted that the primary outcome for inhaled corticosteroids/long-acting $\beta$ agonists (ICS/LABA) given in SMART fashion was superior to lower doses of ICS/LABA given in fixed dose fashion and also superior to fixed dose ICS monotherapy.

We thank Drs Peters, Yan, Reddel and Professor Jenkins ${ }^{1}{ }^{4}$ for highlighting the second relevant Cochrane review. ${ }^{5}$ A thorough reading will reveal that, in the studies examined by Cates and Lasserson, the dosage of maintenance ICS was reduced during the run-in so that, under these conditions, exacerbations requiring oral steroids (but not hospitalisations) occurred less frequently when patients inhaled ICS/LABA rather than short-acting bronchodilator alone. This finding is consistent with our hypothesis that SMART may allow patients to self-treat exacerbations at home without seeking medical care, and begs the question whether it is better to prevent symptoms and exacerbations entirely by adequate amounts of maintenance anti-inflammatory therapy or to rescue patients once symptoms have occurred.

All correspondents appear concerned that, in the table, we displayed only the SMART control outcomes and not outcomes for the comparator limbs. We believe that this was appropriate as our aim was to examine the clinical usefulness of SMART in terms of the control parameters used widely to monitor asthma in the clinical setting. It was not our goal to analyse further the well-known superiority of ICS/LABA to ICS monotherapy or the superiority of higher doses of ICS/LABA to lower doses of ICS/LABA. Bowler and Serisier suggest that the poor control outcomes seen in these trials 'reflects the severity of disease' of participants. ${ }^{6} \mathrm{We}$ respond that the failure of SMART therapy to control severe disease would hardly recommend its use in moderate or mild disease. Indeed, Cates and Lasserson's Cochrane review noted that no superiority was demonstrable with SMART in mild disease. ${ }^{5}$ We must add that it is probably more accurate to describe study participants as having severely uncontrolled disease at recruitment and not necessarily as having severe disease, given that optimal education, compliance and treatment may have controlled their disease.

Reddel and Yan ${ }^{4}$ suggest that our review of SMART results has been selective, a challenge that is difficult to address as we attempted to distil a large body of research literature, analysis and commentary into a review of acceptable length. In our review we acknowledged the well-known and often-emphasised primary outcome of SMART trials, but also attempted to discuss the much less frequently mentioned (and often concealed) effect of SMART therapy on asthma control. We have been chastised for highlighting this outcome and commenting on the dearth of discussion around control, but must note that Bateman and colleagues' manuscript estimating control (on a week-by-week rather than long-term basis) has only recently been published and was available to add to our review only at the galley proof stage of manuscript production. ${ }^{3}$ We did not wish to criticise the use of exacerbations as an end point in asthma trials but wished to point out that, by limiting the choice of primary end point to 'time to severe exacerbation' in all but one SMART trial (which used peak flow), ${ }^{7}$ the body of research has concealed the generally poor asthma control outcomes seen with this strategy. Although we referenced in our review the paper by Kuna and colleagues using double-dummy methodology, we suspect we are not alone in believing that blinding remains difficult and sometimes impossible when inquisitive and observant asthma patients are given 